\title{
Management of an infected graft in the ascending aorta by an omental pedicel graft and irrigation with povidone iodine
}

\author{
William Joyce, Simon Huddy, E E John Smith
}

\begin{abstract}
An infected graft in the ascending aorta was managed by use of an omental pedicel graft and local irrigation with a dilute suspension of aqueous povidone iodine. This conservative approach is an alternative to the high risk procedure of graft replacement. The large abscess cavity associated with the aortic prosthesis was diagnosed by computed tomography.
\end{abstract}

Infection of an aortic prosthesis is a grave complication of cardiovascular surgery. Such infection is associated with a high mortality, limb loss, or both. ${ }^{1}$ Usually the infected graft is removed and blood flow is rerouted through a clean field. This requires technically difficult surgery in very ill patients.

The omentum can 'wall off and localise potentially dangerous inflammation and gastrointestinal perforations. In most individuals the omentum, on a vascular pedicel, is large enough to be used in the thorax. This approach combined with the local antibacterial action of povidone iodine seemed an attractive alternative to replacement of an infected graft.

\section{Case report}

A 65 year old woman was referred with a 3 month history of increasing shortness of breath and a past history of rheumatic fever. On admission she was found to be in acute left FRCSEd, Surgical Professorial Unit, The Mater Hospital, 47 Eccles Street, Dublin 7, Ireland.

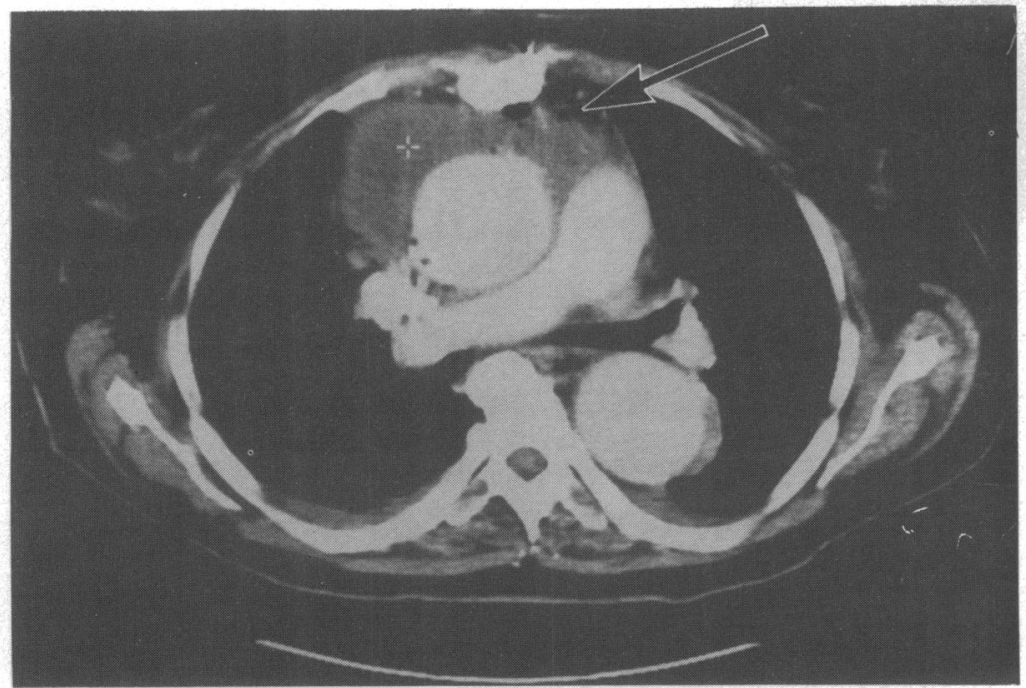

Coronal computed tomogram at $T 5$ level showing a large ( $8 \mathrm{~cm}$ diameter) periprosthetic abscess ( + ) and gas bubbles (arrow) ventricular failure and was initially treated medically. An echocardiogram showed considerable left ventricular dysfunction, severe aortic valve incompetence, and a $10 \mathrm{~cm}$ aneurysm of the aortic root. Subsequent cardiac catheterisation confirmed these findings.

Four days after admission she deteriorated medically and required ventilation. After 48 hours of ventilation and an improvement in her condition she was taken to theatre. After initiation of cardiopulmonary bypass and cooling to $23^{\circ} \mathrm{C}$ centigrade, the aortic valve was replaced by a $21 \mathrm{~mm}$ Björk-Shiley valve. The aortic root aneurysm was resected and replaced with a $34 \mathrm{~mm}$ Gelseal graft.

At first she made a good postoperative recovery. But 3 weeks after operation a fever developed and the sternal wound began to discharge serous fluid. Two of six blood cultures grew a coagulase-negative staphylococcus while the sternal wound grew mixed coliform bacteria only. Treatment with intravenous pivampicillin and cefotaxime was started. Despite a normal white cell count the wound continued to discharge though it remained stable. Computed tomography showed a large abscess cavity associated with the aortic prosthesis. The vascular anastomosis seemed to be intact. She was therefore returned to theatre and the wound was reexplored.

A large periprosthetic abscess cavity was evacuated and the area was washed out thoroughly. The anastomosis was intact. The wound was extended into the upper abdomen. A flap (15 cm long) of greater omentum was mobilised off the transverse colon. This was delivered into the pericardial space through a substernal tunnel and was loosely held in place around the prosthesis. Three drains were inserted into the pericardial space. The wound was closed with sternal wires and deep tension sutures to superficial tissues.

The wound was irrigated postoperatively for 10 days with a dilute aqueous suspension of povidone iodine via the pericardial drains. Culture from the abscess cavity was sterile; none the less, a 6 week course of vancomycin was started. She made an otherwise uneventful postoperative recovery and she was well 18 months later.

\section{Discussion}

Infection of mediastinal aortic prostheses is uncommon but management remains a major surgical problem. Diagnosis can often be dif- 
ficult. In our patient computed tomography established an early diagnosis that led to prompt surgical intervention.

Replacement of the infected graft is associated with a high risk. A more conservative approach by debridement and irrigation with povidone iodine reduces the local bacterial count but generally does not effect a cure. ${ }^{2}$ The major contributing factor to failure in treatment of mediastinal graft infections is the obliteration of the dead space because of the rigidity of the retrosternal compartment. ${ }^{3}$ An omental pedicel graft fills the dead space and reduces the potential for reinfection. ${ }^{4-6}$

As well as having anti-inflammatory properties, the omentum also contains an angiogenesis factor ${ }^{7}$ which encourages new blood supply and provides fibroblasts to enhance healing. This in itself may be of considerable importance in the management of local sepsis. The omentum is large enough to be taken to anywhere in the body to treat graft sepsis.

1 Hargrove W, Edmunds L. Management of infected thoracic aortic prosthetic grafts. Ann Thorac Surg 1984;37:72-7. Edwards M, Richardson D, Klamer T. Management of aortic prosthetic infections. Am J Surg 1988;155:327-30.

3 Mathisen D, Grillo H, Vlahakes G, Daggett W. The omentum in the management of complicated cardiothoracic problems. J Thorac Cardiovasc Surg 1988;95: 77-84.

4 Lee A, Schimart G, Shalkin S. Total excision of the sternum and thoracic pedicle transposition of the greater omentum: useful strategems in managing severe mediastinal infection following open heart surgery. Surgery 1976;80:433-6.

5 Frimpong-Boateng K, Warnecke H, Schuler S. Transposition of the greater omentum for management of mediation of the greater omediation-a case report. J Heart Transplant 1986;5:330-1.

6 Cohen M, Silverman N, Goldfaden D, Levitsky S. Reconstruction of infected median sternotomy wounds. Arch struction of infected
Surg 1987;122:323-7.

7 Goldsmith H, Griffith A, Kupfermqan A, Catsimpoolas $\mathrm{N}$. Lipid angiogenic factor from omentum. JAMA 1984;252:2034-6.

\section{British Cardiac Society}

The Annual Meeting will take place at the Scottish Exhibition Centre, Glasgow on 30 April to 3 May 1991. 